\section{Una invitación a la sociología pública. La investigación social y la precariedad laboral en Chile}

An invitation to public sociology. Social research and precarious labor in Chile

\section{DASTEN JULIÁN*}

\section{Resumen}

El debate sobre las condiciones laborales en Chile cada vez asume mayor protagonismo en la esfera pública. Las políticas laborales parecen ser un tema inevitable para los actores sociales de acuerdo a las condiciones de desigualdad y precariedad que rodean el espacio de trabajo. Ante este escenario, el sindicalismo busca un

Sociólogo. Doctor en Sociología del Trabajo. Académico e Investigador del Departamento de Sociología y Ciencias Políticas.

Facultad de Ciencias Sociales. Universidad Católica de Temuco, Chile. Investigador adjunto del Observatorio Regional UCT. E-mail: djulian@uct.cl reposicionamiento público, principalmente diversificando y modernizando sus tácticas de fortalecimiento y acción. Lo que proponemos en el presente artículo, de acuerdo con este contexto de precariedad laboral, es un debate sobre la sociología, en su expresión activa y pública, y el desafío que enfrenta en la construcción de puentes con la 'sociedad civil', el neoprecariado y el sindicalismo, de forma de jugar un rol activo en la intervención social y obtener un feedback de revitalización y vigorosidad de su salud como disciplina y ciencia en Chile.

Palabras clave: precariedad del trabajo, sociología pública, sindicatos.

\begin{abstract}
The debate about working conditions in Chile is increasingly assuming a greater role in the public sphere. Labour policies inevitably seem to be a topic for social actors according to the conditions of inequality and insecurity surrounding the workspace. In this scenario, the trade union seeks for a public repositioning, mainly diversifying and modernizing its strengthening tactics and action. What we propose in this article, according to this precarious context, is a discussion about sociology, in his active and public expression, and the challenge that it faces in the building of bridges with the 'civil society', the neo-precariat and the trade unionism, as to play an active role in social intervention and get a feedback of revitalization and invigorate its health as a discipline and science in Chile.
\end{abstract}

Keywords: work precariousness, public sociology, unions. 


\section{Introducción}

En los últimos cuatro años, la sociedad chilena ha estado sujeta a una re-emergencia de tensiones y conflictos sociales en materias como educación, salud, medio-ambiente, etc., las cuales constituyen dimensiones sociales que se encuentran sujetas históricamente a la intervención de las políticas neoliberales en el período de la dictadura militar y su continuidad en los gobiernos electos democráticamente hasta la fecha (Salazar 2008; Garretón 2014).

Esta situación, que involucra una serie de consecuencias para el bienestar y la justicia social, han dado paso en la actualidad a la toma de cuerpo, constitución y movilización de diversos actores sociales, quienes se han organizado en pos de reformas estructurales al actual ordenamiento social.

Aun así, pese a la pluralidad de voces y movimientos emergentes, parece ser que los trabajadores, en su acepción clásica (De la Garza 2012, 2005), no han operado de forma sincrónica a estos cambios, especialmente en la rearticulación orgánica con los movimientos sociales y, más bien, han estado en la retaguardia de los procesos de movilización, protesta y reivindicaciones sociales.

Si bien esta dificultad parece estar asociada, por una parte, a la pérdida de una serie de narrativas y tradiciones relacionadas con el movimiento sindical, también es posible reconocer una crisis de la construcción de sentidos colectivos en el trabajo y al proceso histórico de pérdida de autonomía y de poder institucional de las organizaciones sindicales (Aravena y Nuñez 2011; Julián 2014a).
Es posible agregar, como telón de fondo de esta desactivación de las organizaciones de los trabajadores, el proceso ideológico que el neoliberalismo ha impulsado en la construcción discursiva referente a la organización colectiva de los trabajadores (y al sindicalismo). Este proceso se grafica en la redefinición de la posición del sindicalismo en las relaciones laborales y la sociedad en general (Julián 2014a), guiándolo hacia prácticas institucionales, estandarizadas y burocráticas de reivindicación (Gutiérrez 2014).

Sincrónicamente, la sociología y las ciencias sociales se han visto en un proceso que, por medio de una serie de diagnósticos que sin lugar a dudas involucran disposiciones ideológicas y discursivas, ha tendido a desplazar la tematización de fenómenos relacionados al campo propiamente laboral (Garretón 2014; Ramos 2012). Este hecho se ha plasmado en el reemplazo en los estudios referidos de la acción de los trabajadores y sus organizaciones (Góngora, Rodríguez y Leiva 2006; Aravena 2007; Salazar 2009) por aquella de los movimientos sociales.

En el caso de la sociología del trabajo en Chile, esta se ha caracterizado desde la década de los 90's por haber transitado desde los enfoques que estudiaban los procesos de restructuración económica, los cuales se encontraban más cercanos a la economía del trabajo (Agacino 1994; Escobar 2000), hacia el estudio de las consecuencias de la relación asimétrica que caracteriza a las relaciones laborales y la redefinición de la morfología del trabajo en Chile (Díaz et al. 2006; Hernández et al. 2014).

Por su parte, diferentes centros de pensamiento y organizaciones no gubernamentales fueron 
parte estratégica de que los estudios laborales no desaparecieran completamente durante la dictadura militar, mientras que para la fase iniciada en los años 90's fue central el rol del departamento de estudios de la Dirección del Trabajo en un enfoque neoinstitucional y de la regulación. De la misma forma, el retorno al país de varios/as investigadores/as que se encontraban en el exilio, puso en diálogo las tendencias internacionales de los estudios del trabajo con la nueva realidad chilena, concentrando su acervo temático en las características del sindicalismo, el mercado laboral, la informalidad y el desempleo en la estructura ocupacional.

Reconociendo la importancia de las políticas neoliberales en el trabajo y el empleo, la sociología del trabajo se concentró en identificar los procesos macro-sociales, las políticas sociales y la redefinición de los espacios de trabajo, por medio de la producción de distintos enfoques de investigación (Cárdenas 2005; Aravena 2007; Sisto 2009; Aravena y Núñez 2011; Salas 2013). Aparecieron estudios sobre las transformaciones en el trabajo, las desigualdades de género, el proceso de precarización laboral, la transnacionalización económica, el análisis de las políticas de flexibilidad laboral y la emergencia de la subcontratación. Todos ellos procesos que se ponían en comunicación con enfoques propios de la teoría social, la economía política y la psicología social (Díaz et al. 2006; Soto 2008; Caro 2012; Stecher y Godoy 2014).

En este debate, también resultó importante la configuración de 'nuevos sujetos' e identidades laborales, ya que el contexto de "crisis del neoliberalismo", ha tenido evidente impacto en la forma de entender y "vivir el trabajo"
(Soto 2008), así como de forjar un núcleo de integración inestable por medio del empleo (Julián 2014b). De esta forma, la sociología del trabajo ha dado paso a investigaciones y exploraciones novedosas de los fenómenos de la precariedad, las subjetividades laborales y los cambios en los repertorios de acción sindical (Caro 2012; Sisto 2012; Stecher 2013; Hernández et al. 2014; Soto 2014), centrándose en un posicionamiento crítico a la situación y las consecuencias (subjetivas, intersubjetivas y objetivas) de trabajar hoy en Chile (Sisto, 2009; Stecher y Godoy 2014; Soto 2014).

A partir de lo anterior, entendemos que existen en la actualidad distintas perspectivas críticas que apuntan en la dirección de reivindicar 'el trabajo' como un espacio en disputa, en contradicción y en tensión respecto al poder político-económico. Pese a ello, creemos que esta diversidad de posiciones aún no se expresa en un programa de investigación compartido y/o en la conformación de un debate sociológico profundo y relacional sobre el significado de las transformaciones del trabajo y el posicionamiento de la sociología (del trabajo) en esta materia.

Porello, en este artículo revisaremos la propuesta presentada en el enfoque de la llamada por Michael Burawoy (2012, 2005) "sociología pública", ya que vemos en esta propuesta un puente de comunicación en el debate de las ciencias sociales, la sociología del trabajo y la 'sociedad civil', con miras al fortalecimiento de 'un público' (en conformación). Para ello, consideramos el fenómeno de la extensión de la precariedad laboral y la constitución de un neo-precariado en Chile (Julián 2014b) como elementos centrales de diálogo e interpelación. 
En la primera parte de este artículo, hacemos una revisión del concepto de "sociología pública" presentada por Michael Burawoy (2014, 2012, 2005), para dar un alcance teórico de lo que significa la práctica académica y científica desde un enfoque crítico, en escenarios de alta complejidad institucional. Luego, ponemos en dialogo esta 'visión pública' de la sociología, con los escenarios de precariedad laboral y el neoprecariado, como sujeto colectivo (Garretón 2014), pensando en la interrogantes y potencialidades que este caso envuelve para la sociología y la investigación social. Finalmente, del debate anterior desprendemos a modo de conclusión la interpelación al investigador social referente a la triangulación entre teoría, epistemología y metodología hacia el modelo de investigaciónacción en el espacio laboral y sindical.

\section{La sociología pública ¿sociología para quién y para qué?}

En las últimas décadas, las disciplinas de las ciencias sociales han atravesado un proceso de profunda institucionalización (Wallerstein 2006; Garretón 2014), especialización (Burawoy 2005; Ramos et al. 2008) y profesionalización (Burawoy 2012, 2003). Las políticas neoliberales han empujado los procesos hacia modelos de estandarización, positivización, normalización y mercantilización de las ciencias sociales, las cuales han encontrado contadas respuestas dentro del campo académico y social (Kalleberg 2005, 2000; Ariztía y Bernasconi 2012; Ramos 2012) y, claramente, han impactado en el desarrollo de la sociología.

De acuerdo con este contexto, Michael Burawoy $(2014,2012,2005,2003)$ ha sido uno de los promotores de las actuales reflexiones sobre la salud de la sociología como disciplina científica y social. Haciendo una clara referencia a Walter Benjamin, Burawoy (2005: 199) señala que "en sus inicios la sociología aspiraba a ser como el ángel de la historia, buscar orden en los fragmentos rotos de la modernidad, tratar de salvar la promesa del progreso", mientras que en la actualidad el panorama a nivel institucional y de normalización de la sociología parece reconocer 'su progreso' en:

\footnotetext{
"una batería de técnicas disciplinarias -asignaturas estandarizadas, bibliografías normalizadas, clasificaciones burocratizadas, exámenes continuos, reseñas de la literatura, tesis doctorales a medida, publicaciones evaluadas, el todopoderoso CV, búsqueda de trabajo, estabilización laboral y posterior politización de los colegas y de los sucesores" (Burawoy 2005: 199-200).
}

Este contexto de profunda institucionalización marca una esfera de auto-reproducción de la sociología en la formulación de sus propios criterios de producción, lo cual debilita la potencia efectiva y eficiente de la disciplina, paradójicamente, en la misma sociedad (Garretón 2014). Y es que en el caso de la sociología la validación del conocimiento producido se encuentra bajo una disyuntiva. Como señala Maribel Nuñez (2011), esta validación:

\footnotetext{
"resulta sólo del debate y la construcción de acuerdos intersubjetivos de una comunidad de especialistas; o bien, su pertinencia científica tiene alguna relación con la eficacia con la que ofrece recursos conceptuales y cursos de acción coherentes y viables para que los distintos sectores de la sociedad puedan perseguir sus intereses" (Nuñez 2011: 235).
}

Como lo reconocen Ariztía y Bernasconi (2012: 133), el diagnóstico de Burawoy en la reunión anual de la Asociación Norteamericana de Sociología el año 2004, fue que la sociología se encontraba "dominada por el quehacer profesional y académico", y que a partir de ello, "enfrentaba un déficit en su intención crítica y 
en su capacidad de proveer de explicaciones comprehensivas de los asuntos sociales". Es decir, se había producido un consenso tácito que optaba por un nivel de validación socio-técnico y profesional del conocimiento producido por la sociología, lo cual cerraba y estrechaba los límites de la crítica al interior de la disciplina.

Ante este mapa de constricciones y presiones para la "vocación crítica" de la sociología, es que Burawoy propone el proyecto de la sociología Pública como parte de una

\begin{abstract}
"re-traducción sistemática de nuestra disciplina, esto es, devolver el conocimiento a sus inspiradores haciendo públicas las cuestiones referentes a problemas privados y así regenerar la fibra moral de la sociología" (Burawoy 2005: 200).
\end{abstract}

La metodología de trabajo, si podemos Ilamarla así, está sentada en la promoción epistémica de una "multiplicidad de sociologías públicas" (Burawoy 2005: 202), es decir, una concepción que involucra concentrarse en la relación intersubjetiva establecida y a establecer entre la(s) sociología(s) con los diversos públicos, a la vez que "se trata de investigar cómo se produce esa conversación" (Burawoy 2005: 202).

A este proyecto le acompaña un contexto marcado por la paradójica situación de desenvolverse en un "régimen que es profundamente anti-sociológico en su ethos y hostil ante la propia idea de «sociedad»" (Burawoy 2005: 201), lo cual involucra directamente los desafíos de legitimación e impacto de la disciplina sociológica. Esta paradoja expresada por Burawoy reconoce que es la sociedad la que crea las demandas por una sociología pública, mientras que, por otra parte, es la misma sociedad la que genera los obstáculos e impedimentos para su desarrollo y "producción de lo social" (Ariztía y Bernasconi 2012).
Aún así, en esta obvia dificultad relacional del campo de emergencia de la sociología pública, Burawoy (2003) tenderá a pensar, de manera evidentemente neo-gramsciana, que la forma de establecer la relación con 'el público' (de la 'intelectualidad') es lo que de cierta manera vitalizará el 'proyecto orgánico' de la sociología pública. Esta revitalización se produciría por medio de una relación en la que "el sociólogo trabaja en estrecha conexión con un público visible, denso, activo, local y a menudo a contracorriente", donde el 'hacer sociología' tiene como objetivo principal "hacer visible lo invisible, hacer público lo privado" (Burawoy 2005: 203).

Para ello, la sociología pública debe considerar 'el hacer' de la sociología profesional, la cual suministra "los métodos adecuados y ya experimentados, los cuerpos de conocimiento ya acumulados, las orientaciones necesarias y los marcos conceptuales" (Burawoy 2005: 205); y/o lo que Wallerstein reconocerá como la imperiosa necesidad de "obtener la legitimación pública" (2006: 80), en referencia crítica al proceso de separación entre investigación y enseñanza (en el sistema universitario), de forma de "contar con las bases materiales necesarias para sostener la investigación académica" (Wallerstein 2006: 80).

La propuesta de Burawoy está claramente vinculada a un diagnóstico realizado en las sociedades del Norte (Ariztía y Bernasconi 2012), y en referencia a un campo amplio de comprensión de la sociología que abarca diferentes subdisciplinas y, por lo mismo, involucra la imagen y complejidad de diferentes públicos en referencia a las sociedades del sur global (Prashad 2013; Burawoy 2014). Aun así, este proyecto muestra una alta potencialidad como herramienta de 
focalización y producción para contextos como el latinoamericano, donde la sociología y el pensamiento social guardan una larga tradición de carácter público y crítico (Zemelman 2002, 1995; Marini 2007; González 2009).

En nuestro caso particular, resulta atingente preguntarse por cómo es posible insertar este proyecto de hacer sociología, cuando el contexto que rodea y condiciona el contenido público de la sociología se encuentra atravesado por la configuración y conformación de las sociedades modeladas por el neoliberalismo y el neocolonialismo (Ariztía y Bernasconi 2012). Mientras que en el caso específico de la sociología del trabajo, como especialidad referente a las problemáticas y el fenómeno del trabajo en América Latina, cabe cuestionarse cómo puede este proyecto desarrollarse en un contexto donde prima la precariedad laboral, el debilitamiento de los actores colectivos y la vulnerabilidad de los derechos sociales y laborales (De la Garza 2005; Braga et al. 2008; Weller 2011; Burawoy 2014).

Es claro que el proceso de expansión de la precariedad laboral y la vulnerabilidad social, no es una situación propia de la sociedad chilena, sino que, más bien, se encuentra enlazada al actual desarrollo del panorama social latinoamericano. Su especificidad ha sido resaltada, desde la sociología del trabajo latinoamericana, como un componente propio de los procesos de neoliberalización (Barattini 2009), la ampliación del trabajo no clásico (De la Garza 2012), la formación de identidades precarias (Battistini 2009), la morfología del trabajo (Antúnes y Pochmann 2008; Antúnes 2011) y la heterogeneidad ocupacional en la región (Guadamarra et al. 2012).

Desde la materialidad de la precariedad laboral y sus consecuencias en la vida cotidiana, es que la sociología del trabajo se ha acercado a un diálogo con la teoría social latinoamericana (De la Garza 2005, 2001; Antúnes 2003; Julián 2014c), centrando su análisis en los procesos de modernización y modernidad periférica, la conformación de subjetividades, las tesis de la marginalidad y la informalidad laboral, así como los cambios en el modelo de acumulación de capital (Sotelo 2003; Antúnes 2005; Neffa 2009).

Dichos procesos dan cuenta de un escenario contradictorio y crítico, considerando que la precariedad, en su significación política, es el camino a la problematización por parte de la 'sociedad civil' de una 'cuestión social' ante la hegemonía y avance de las políticas neoliberales (Barattini 2009; Battistini 2009; Julián 2013). Esta problematización se desarrolla en contextos donde históricamente la precariedad se ha instalado como una condición de la reproducción social de las sociedades dependientes (Marini 2007; Julián 2014c).

Este último hecho significa entender la precariedad laboral como una condición relacional e histórica, en cuanto:

1) Al carácter que posee este fenómeno en el ejercicio de derechos políticos, civiles, laborales, sociales etc., su estrecho vínculo con la formulación de las políticas de aseguramiento, la promoción de condiciones de protección social y los mecanismos de integración y cohesión social (Castel 2010b: 189206). Este escenario se encuentra atravesado por las expectativas y demandas de distintos actores por el bienestar y equidad social, en el marco de "un pacto por la igualdad y la cohesión social” (Garretón 2014: 104-109); y

2) Al debate que ha desarrollado la sociología y el pensamiento social latinoamericano sobre las condiciones y relaciones específicas de poder, desigualdad, discriminación, racismo, (neo) colonialidad, clasismo, machismo, marginalidad y (sobre) explotación, que han dado forma a las 
sociedades latinoamericanas (Marini 2007; González 2009), así como a las posibilidades de su subversión (Zemelman 2002, 1995; Fals Borda 2009).

Por ello, entendemos 'la precariedad' como un desafío central para la investigación social, lo cual implica el ejercicio sociológico de reconocer 'las bases materiales' de este proceso, en el decir de Wallerstein, con las cuales la sociología pública debiese contar para la definición de su(s) público(s). Estas bases materiales, en el caso específico de la precariedad laboral, creemos que podrían ser identificadas en la conformación, condición y expansión del 'precariado' como sujeto-objeto de las transformaciones del trabajo, y en la compleja (re)producción socio-histórica de una heterogeneidad de escenarios laborales a nivel global, regional y local (Dörre 2009; Standing 2011; van der Linden 2014; Julián 2014c).

De esta forma, apuntamos a la articulación del fenómeno de la precariedad laboral con un análisis y generación de información que permitan visualizar públicamente los procesos que los y las trabajadoras deben 'experienciar' actualmente. Para ello, sabemos que no basta una constatación discursiva de la cuestión, sino que se hace necesario poseer una clara y rigurosa definición acerca de lo que entenderemos por 'precariado' y por 'precariedad laboral', así como sus significados, contradicciones y problemáticas para el ejercicio de la sociología y la investigación social.

\section{Precariado y precariedad: Un 'nuevo público' para la sociología}

La precariedad, como condición políticamente inducida, implica una compleja red de disposiciones a la subjetividad y la subjetivación, y la diversificación de modelos de sujeción y (re)producción social, así como también la conformación de narrativas, posiciones de enunciación, emociones y discursos en su apropiación simbólica, significación y en la (re) constitución de imaginarios sociales (Dörre 2009; Julián 2013).

Desde esta complejidad es que la precariedad involucra la conformación de sujetos y la construcción de subjetividades que pueden compartir o no una identificación con la condición de precariedad, como reconocerse intersubjetivamente y suponer acciones colectivas frente a esta condición (Battistini 2009; Julián 2013). Este hecho supone la figuración de 'un público' específico para la sociología, el cual puede ser pensado como una comunidad provisional formada "en respuesta a temas de su preocupación, que existiendo las instituciones y los procedimientos son incapaces de manejar" (Eyal 2013: 20). A este público particular le llamamos 'el precariado'.

Una de las definiciones más populares del precariado es posible encontrarla en los textos de Guy Standing (2011), quien señala que el precariado es un conjunto de trabajadores que sostiene "mínimas relaciones de confianza con el capital o con el Estado, [sin la seguridad de] contratos de largo término y la ausencia de protección en contra de la pérdida de empleo" (Standing 2011: 8-9), desarrollando situaciones de inestable cohesión para la sociedad, por medio de la persistencia decondiciones limitadas de bienestar, protección y aseguramiento social (Castel 2010b; Neffa 2010).

A este proceso de precarización descrito por Standing -y por vasta literatura y autores en el tema-, se sumaría una creciente vulnerabilidad 
estructural que (re)dinamiza las relaciones entre las clases sociales, reconfigurando los escenarios de problematización, de la llamada por Pierre Bourdieu (1999) "situación" y "condición de clase", especialmente desde el campo del poder y la conformación de los actores colectivos en el trabajo (Díaz-Salazar 2004; Kalleberg 2009; Burawoy, 2014).

Mientras que la definición anterior del precariado se enmarca en el caso de las sociedades del Norte, en el caso de la realidad del Sur Global, Ricardo Antúnes (2011: 113) señala que "si bien las formas de la restructuración productiva han sido diferentes, un rasgo prácticamente constante, cuando se observa la realidad cotidiana del trabajo, fue la tendencia a un aumento de los mecanismos de desregulación y a la propia precarización de la fuerza de trabajo".

Este pilar de confluencia entre desregulación y precarización, es el que constituye y da cierta homogeneidad a la hora de entender la introducción de mecanismos de pauperización del empleo en América Latina por medio de las políticas neoliberales en las décadas de los 80's y 90's (Sotelo 2003; Bensusán 2009; Neffa 2010; Weller 2011; De la Garza 2012), lo cual figura las tendencias hacia:

a) La conformación de un neo-precariado: caracterizado principalmente por la redefinición de la composición de la clase trabajadora, la transformación de la estructura productiva, la ampliación del sector servicios, las tensiones entre la 'modernización productiva' y descomposición de la agricultura, la inserción laboral precaria de jóvenes y mujeres, la extensión de la informalidad laboral, la marginalidad social, la pobreza, etc.; y

b) La consolidación de la condición histórica de precarización y subvalorización de la fuerza de trabajo en las sociedades postcoloniales: este fenómeno tendría relación con los procesos de relocalización de la producción y la financiarización a nivel global, los cambios en las matrices políticas, la individualización y disciplinamiento laboral vía mecanismos legalesformales, y la nueva relación entre las clases sociales.

Ambas tendencias supondrán paradójicamente para los trabajadores, de manera general, el desafío colectivo y la exigencia objetiva de

\begin{abstract}
"impedir la deconstrucción de los derechos sociales y obstaculizar la expansión de las formas diferenciadas de precarización del trabajo, frenando la deconstrucción del trabajo realizada por el capital y recuperando y/o creando una nueva forma de sociabilidad del trabajo efectivamente dotada de sentido" (Antúnes y Pochmann 2008: 199).
\end{abstract}

Pero ¿quién es este neo-precariado y cuáles son sus posibilidades colectivas reales de acción ante este proceso de precarización? Desde nuestra perspectiva, el neo-precariado es un complejo y disperso entramado de sujetos en la estructura productiva, presente en una extensa y diversa red de escenarios laborales (Soto 2014). El neo-precariado se define, por una parte, por condiciones salariales de insuficiencia, relaciones contractuales inestables, flexibles y/o inexistentes, y condiciones laborales que rondan en la inseguridad y la desprotección social; mientras que, por otra parte, se caracteriza por la heterogeneidad de sectores productivos, espacios laborales, cualificaciones y ocupaciones en las cuales se presentan inserciones laborales 'atípicas', sumado a una alta vulnerabilidad social (Castel 2010a; Neffa 2010; Julián 2014b).

En neo-precariado es una combinación de estos elementos, principalmente de diversas dimensiones, tales como la desprotección de los sistemas de formalización en salud y previsión social, la inestabilidad y la incertidumbre propia de la flexibilidad laboral (contractual, salarial, organizacional y funcional). A ello podemos agregar las condiciones de trabajo 
ergonómicamente poco apropiadas y de riesgo e inseguridad vital, y la insuficiencia salarial como un mecanismo de disciplinamiento y pauperización en la valorización de la fuerza de trabajo (Julián 2014b).

El carácter de 'neo' supone un reconocimiento a la precariedad como una condición histórica del trabajo en América Latina. Por ello, lo 'neo' se encuentra vinculado a las transformaciones productivas a escala global desde la década de los 80 's, con un cambio en la matriz social y de desarrollo (Blanco y Julián 2014; Gago y Mezzadra 2015). A la vez, se relaciona a la emergencia de proyectos político-sociales fundados en principios y expectativas civiles como la equidad, justicia y democracia desde el postneoliberalismo (Salazar 2009; Mayol 2013; Julián 2014b).

De todas formas, este acercamiento a la precariedad laboral exhibe una comunicación y reconocimiento de los problemas históricos de la sobreexplotación de la fuerza de trabajo en el llamado "tercer mundo" (Sotelo 2003; Ross 2008), las contradicciones de las modernidades periféricas (Lechner 1990), los cambios y procesos en curso en la heterogeneización de la clase trabajadora (Antúnes 2003; 2011; Guadamarra et al. 2012), y la nueva dinámica de movilización y reactivación sindical (Julián 2014a).

Este último punto es muy importante, ya que, como es reconocido en el plano de las relaciones laborales y el espacio de trabajo, la representación institucionalizada de los intereses de los trabajadores sigue atada al desenvolvimiento de los actores sindicales y de su reconocimiento legal (Aravena 2007; Aravena y Nuñez 2011). En el caso de Chile, las formas y expresiones que ha cobrado este neoprecariado se encuentran lejanas de hallar una sincronización y la activación de un grupo sólido y cohesionado de movilización y reivindicación política, en términos de organización y acción colectiva, debido, en gran parte, a la misma obstaculización que representa la precariedad laboral para la asociatividad (Díaz-Salazar 2004; Battistini 2009; Julián 2013).

En esta diatriba de imposibilidades de reconocimiento y acción en el mismo seno de una heterogenia y fragmentada clase trabajadora, el precariado ha cobrado expresiones en la adhesión a conflictos políticos y sociales que, paradójicamente, alejan al trabajo como eje político. Su rudimentaria y emergente explosión en el escenario público no ha logrado identificar claramente a la precariedad laboral como un foco de activación reivindicativa e identidad colectiva y, más bien, ha actuado de manera dispersa y descentralizada en diversos conflictos ocupacionales, sectoriales, regionales $\mathrm{y} / \mathrm{o}$ locales.

Por su parte, la precariedad (laboral) se expresa como un fenómeno estructural, al cual le sucede una lógica de desbordar las distintas relaciones del campo social y la vida cotidiana, ya que cuenta con la propiedad de transitar intermitentemente impregnando cada una de las decisiones, esferas y relaciones de poder subyacentes a la decisión del Estado y su institucionalidad (Bessant 2008; Castel 2010b). De la misma forma, la precariedad se ha institucionalizado (Julián 2014b), desarticulando solidaridades y disponiendo disciplinarmente los espacios de trabajo.

Centrándonos en la materialidad del contexto social y retomando la tradición del pensamiento 
latinoamericano, la precariedad laboral como eje para una sociología pública podría ser pensada como un espacio de conversación y diálogo entre dos esferas sociales: primero, como un diálogo entre distintas corrientes críticas y de propuestas de análisis diversas del capitalismo periférico en la esfera académica; y, segundo, un diálogo desde la potencialidad de los imaginarios políticos y públicos de los sujetos subalternos en América Latina, desde la esfera política. Este diálogo solo es posible debido al carácter residual y sintomático que caracteriza a la precariedad y a su trazabilidad en la construcción de las sociedades latinoamericanas.

Reconociendo este carácter de la precariedad laboral, creemos que las preguntas a dialogar para un proyecto de investigación formulado desde la sociología pública vendrían a ser: ¿Cuáles son las problemáticas que puede visualizar la sociología pública en esta tendencia a la precarización laboral y social? ¿Y cuáles son los medios y acciones necesarias de emprender con el fin de fortalecer su proyecto y práctica investigativa desde el sujeto y su público?

\section{Pensar públicamente la precariedad laboral}

Para la propuesta de Burawoy (2005), claramente este proceso dificultoso de constitución real de una audiencia cohesionada en el espacio del trabajo, moviliza el conocimiento reflexivo a la interrogante sobre el "valor de las premisas de la sociedad así como de nuestra profesión" (Burawoy 2005: 206), interrogando el proyecto de la sociología crítica con el compromiso con las audiencias extraacadémicas. Entonces, en términos concretos ¿qué puede hacer la sociología pública en un contexto de precariedad laboral?
De la misma forma que en la precariedad, como categoría y producto social, no sería una característica que pretenda homogeneizar la producción científica de la sociología del trabajo, debiésemos interrogar nuestra práctica investigativa desde la diversificación y complejidad de sus expresiones. Al constituir la precariedad un fenómeno espacio-simbólicotemporal (Julián 2014c), el cual permite captar la dimensión socio-histórica de la construcción de 'un público', un contexto y un sujeto, la práctica sociológica de investigar y generar conocimiento se adecua y sujeta, directa o indirectamente, en referencia a este mismo escenario del cual la precariedad es parte ${ }^{1}$.

Considerando lo anterior, reconocemos dos puntos centrales como supuestos epistemológicos para la generación de una propuesta de investigación que entreteja la relación existente entre sociología pública y un público como el neo-precariado:

1) La producción de conocimiento está asediada por la misma lógica racionalizadora funcional que moviliza la precariedad: Si entendemos a completitud la dimensión propuesta por la "producción de lo social" desde la sociología (Horkheimer 1998: 43-49; Burawoy 1990; Ariztía y Bernasconi 2012), podremos entender que la funcionalidad ideológica no permite la diferenciación del sistema ciencia del modelo económico, y profundiza la lógica que Burawoy llama el 'conocimiento técnico', lo cual impregna a las estructuras y organizaciones con miras a reproducir este fenómeno de forma sistémica, burocratizando la producción científica y la profesión sociológica (Zemelman 1995; Burawoy 2005; Sisto 2005; Bessant 2008; Bourdieu 2008).

Sin lugar a dudas, consideramos que este puede ser un debate más extenso de intercomunicación entre la investigación social del trabajo hoy en día en Chile y América Latina. Lejos de una pretensión normativa de hegemonizar la producción teórica e investigativa, resaltamos la idea de un diálogo con diversos enfoques y matrices teóricas del trabajo, a partir del carácter sintomático de la precariedad laboral. 
2) La espacialidad y temporalidad de la precariedad hace que cada uno de estos procesos sea profundamente dialéctico y diferenciado en su expresión global, continental, regional, nacional y local: Los sujetos del trabajo, y los sentidos del trabajo (Antúnes 2005) generan el carácter epistemológico socio-histórico diverso de la sociología pública. Ello invita a la sociología a trabajar con poblaciones heterónomas y heterogéneas, así como a desarrollar premisas reconstructivas y críticas de un universalismo (del trabajo), pensando desde la pluralidad de alteridades, la memoria histórica y los proyectos políticos emergentes de los sujetos subalternos en diversos contextos sociales (Van der Linden 2014; Julián 2014c; Gago y Mezzadra 2015).

Considerando estos dos puntos, la sociología pública asume el desafío, como proyecto de investigaciónsocial, de interactuary desarrollarse en un contexto de extendida profesionalización y tecnificación de la sociología. A la vez, se propone generar sus propias categorías en contextos marcados por interrelaciones globales que tienden a la abstracción de las realidades locales. Esta situación se grafica, especialmente, en referencia a los residuos epistemológicos eurocéntricos y universalistas que no consideran una "interacción mundial" (Wallerstein 2006: 83) de las ciencias sociales.

Desde esta perspectiva, también se encuentra presente el problema de la precariedad como "fetiche" (Van der Linden 2014), en cuanto 'categoría aislada' de su marco de producción. Para la sociología pública, esta situación implica pensar los 'contextos de precariedad' como formas concretas de expresión y ejercicio de relaciones de poder, donde la articulación de los actores sociales -en la multiplicidad de las contradicciones entre género, clase, territorio, etnia y cultura(s)- juega un rol central para entender las dinámicas de compromiso, imposición y sujeción propias de los regímenes de valorización y (re)producción del capital (Ross 2008; Dörre 2009; Kalleberg 2011, 2009).
Así es como, para el caso de Chile, la sociología pública no tendría otra posibilidad que recurrir primeramente al análisis de la implementación del neoliberalismo como inducción a la precariedad. Como es reconocido por distintos autores (Moulián 1997; Mayol 2013; Garretón 2014), el neoliberalismo jugó un rol central en la modelación de un régimen disciplinario y la reproducción de las relaciones de dependencia entre salario y reproducción vital en las últimas tres décadas (Blanco y Julián 2014; Julián 2014b). A la vez, este proceso tuvo implicancias "sobre la relación entre sociología y sociedad, especialmente durante los años noventa, cuando se recomponía la conexión entre los intelectuales y la sociedad luego del quiebre democrático de 1973" (Ariztía y Bernasconi 2012: 136).

Por otra parte, uno de los desafíos que encuentra la sociología pública es el generar una arqueología identitaria y de identificaciones sociales vinculadas al fenómeno de la precariedad laboral en su proceso de mutación y constitución en el escenario del trabajo (Battistini 2009). De la misma forma se le presenta el desafío de articular este contexto de precariedad con su extensión a una interrelación global y social, con por ejemplo, la categoría de "sur global" (Prashad 2013; Munck 2013; van der Linden 2014), y de introducir este debate como parte de un proceso histórico de transformaciones sociales, culturales y económicas.

Por otra parte, se extiende el 'desafío práctico' de contar con información operacionalizada y fuentes estadísticas que entreguen un panorama general de la situación de precariedad. Esto debiese ser complementado con una profundización cualitativa en el tema, con el objetivo de dar cuenta del nuevo público y de la emergencia y expresión del precariado, a partir 
de, por ejemplo, su influencia en las estructuras sindicales y el mundo del trabajo.

Este elemento práctico de la sociología se encontraría enlazado a la necesidad de un 'conocimiento reflexivo', fruto de un diálogo fluido y continuo 'interesado en los fines'. Este diálogo, en el decir de Burawoy (2005: 206), debiese tomar lugar "dentro de la comunidad académica" para abordar "los fundamentos de sus programas de investigación" que esta misma comunidad sostiene, "o entre académicos y los diferentes públicos en torno a la dirección de la sociedad".

De allí que la sociología pública en Chile, orientada a los contextos de precariedad, requiere una interpelación e interrogación acerca del carácter de el/los 'público(s)' de la sociología en referencia a dos cuestiones: ¿para qué el conocimiento?, y ¿para quién? Estas preguntas, de acuerdo a Burawoy (2005), forman un núcleo de disenso con la propuesta de Wallerstein (2006), en cuanto a lo que Burawoy entenderá como la labor de visibilizar y movilizar la investigación social hacia los problemas de la esfera pública.

Por lo tanto, desde el enfoque de la sociología pública y en el caso particular chileno, creemos que se hace necesario considerar el actual rol que desempeña la 'sociedad civil' y/o las clases sociales en la redefinición de las políticas de la esfera pública, y la movilización de contenidos temáticos a que han sujetado el campo político en los últimos años.

Este conjunto activo y dinámico de conformación de actores colectivos, de demandas, expectativas, proyectos y exigencias, debe ser parte fundamental de reflexión y 'visibilización' en la investigación social, lo cual -desde el punto de vista de la sociología pública- debe estar acom- pañado de 'profesionalidad', como imperativo técnico-normativo que promueven las instituciones académicas y la institucionalización de la profesión sociológica (Bourdieu 2008; Burawoy 2012).

Innegablemente, a este 'optimismo de la voluntad' debe acompañarle dialécticamente 'el pesimismo de la inteligencia', siempre, en palabras de Antonio Gramsci, contra la pasividad intelectual-política y el 'escepticismo del porvenir'. Por ello, la sociología pública no debiese dejar de cuestionarse en su ejercicio si será posible su tarea en contextos de amplia mercantilización, precariedad y precarización social, así como si hay algún ejemplo que pueda orientar esta tarea en la actualidad y si existe realmente desde las ciencias sociales en Chile la intención de orientar su producción hacia un foco público de visualización de temáticas como la precariedad laboral.

De todas formas, este tema y esta reflexión que apuntan críticamente al escepticismo y 'la pesadez intelectual' constituyen parte de un nuevo artículo, el cual tenga como objetivo discutir las políticas de 'modernización de las relaciones laborales', la confluencia entre académicos y los actores sociales, sus horizontes políticocolectivos y la producción de una agenda de investigación desde el enfoque de la sociología pública. A la par, es necesario desarrollar experiencias y espacios concretos de acción para las expectativas de los actores sociales respecto a las interrogantes que presenta la precariedad laboral y su debate público en la actualidad.

\section{Conclusiones}

La problemática expuesta en este artículo, presentó las potencialidades de compenetración 
entre los investigadores sociales, en su acercamiento a las problemáticas del mundo laboral, y la ampliación de "lo laboral" a "lo social" (De la Garza 2005). Nuestra reflexión se concentró en esta compenetración a partir del ejercicio de dinámicas que potencien socialmente la actividad científica de la investigación social y fortalezcan la posición de los actores mayormente vulnerados en el modelo de valorización precario de trabajo en Chile, es decir, los trabajadores.

Más específico aun, la articulación de tres dimensiones tales como el (neo)precariado, la precariedad laboral y la sociología pública exhibe la idea de repensar estrategias de investigación con métodos de intervención social y una democratización en la difusión del conocimiento producido, pensando en 'la sociología como un deporte de batalla', que medie humildemente en la organización y el fortalecimiento de la sociedad civil (Barchiesi 2011; Boltanski 2011; Burawoy 2014).

He allí la potencialidad de la cual hablamos. Esta se proyecta ante el explosivo crecimiento del fenómeno de la precariedad laboral a nivel global y nacional (Ross 2008; Barattini 2009) y regional (Weller 2011; Guadamarra et al. 2012; Julián 2014b), lo cual involucra una forma concreta de desarrollar un acercamiento entre la Investigación social, la sociología del trabajo y los desafíos que encuentran los trabajadores en este precarizado mundo del trabajo y las relaciones laborales.

Esta actual problemática supone un doble desafío intelectual para la sociología pública y las ciencias sociales:

a) Un desafío intelectual académico: una visibilización de las condiciones de organización, los espacios de sujeción, la estructuración de acciones colectivas y las formas de reconocimiento que emergen en los espacios de trabajo puestas en relación con el debate de la precariedad (laboral). A la vez, generaría un 'vaso comunicante' para la comunidad académica de la sociología del trabajo, con el fin de reconocer la heterogeneidad de sus espacios, relaciones y sujetos de estudio con otros investigadores/as en contextos similares con amplia información y producción científica. Finalmente, comprender este conjunto de elementos se vuelve central para el análisis de las nuevas formas y las tensiones que atraviesa el sindicalismo y la clase trabajadora en la actualidad, en su permanente amenaza de crisis, inmovilidad y extinción en el predominio de la política neoliberal del trabajo (Bourdieu 1999; Braga et al. 2008).

b) Un desafío intelectual orgánico: una participación e involucramiento activo y crítico en el proceso de 'politización' del trabajo, 'modernización' de las relaciones laborales y de las lógicas de gestión y democratización que involucra 'el trabajo' en Chile. De esta forma, resulta necesario que la investigación avance desde la constatación descriptiva de la precariedad a interesarse por el rol de las organizaciones sindicales, el apoyo asociativo a las identidades colectivas y laborales, sumado a la proyección de sus bases organizativas hacia el espacio de la 'sociedad civil' (Cortina 1997; Burawoy 2005). Ello implica reconocer las nuevas lógicas, repertorios y tácticas de información, difusión y de influencia en la opinión pública y el sistema político que la clase trabajadora desarrolla en los contextos de precariedad.

De estos desafíos se desprende que una sociología pública que se oriente hacia el espacio laboral, debiese tener un componente activo en la capacidad de socializar información acerca de procesos y experiencias de innovación, fortalecimiento organizacional, comunicacional y estructural de los sindicatos. Pero también no debiese olvidar la existencia de otras organizaciones de trabajadores/as y su relación con los movimientos sociales, donde la precariedad social es un elemento, síntoma y/o fractura de la matriz de reproducción social (Blanco y Julián 2014), así como un nuevo escenario para desafiar la asociatividad y el bienestar de los sujetos (Díaz-Salazar 2004; Braga et al. 2008; Barattini 2009). 
Desde nuestra experiencia de investigación, podemos agregar que el proceso de significación del trabajo, en el caso del discurso de los dirigentes sindicales del país, no es homogéneo ni cohesionado respecto a la precariedad laboral. Aun así, ha articulado redefiniciones de manera emergente y colectiva de conceptos (o bienes simbólicos) como 'dignidad', 'justicia', 'igualdad', etc., exhibiendo las bases de un ethos reconstructivo de la condición laboral, de re-valoración del trabajo y re-significación de sí mismos (Julián 2014a; 2014b; 2014c).

Esta situación, sumada a un debate emergente acerca de la 'cuestión laboral', ha puesto en tensión a los actores del sistema político y económico que anteriormente sujetaban la asimetría de las relaciones laborales y el incumplimiento de convenios internacionales
(Convenio No. 87 y No. 98 de la OIT), en el ejercicio ideológico del 'no-hay-ideología' en las soluciones socio-técnicas al neoliberalismo, naturalizando y normalizando la precariedad laboral.

Este escenario se conforma en un proceso que tiende a redefinir el principal punto de vista del ejercicio sociológico en búsqueda de la salud de la 'sociedad civil' (Burawoy 2014, 2005) y de una politización del trabajo (Julián 2014a). En este sentido, creemos que este artículo forja una invitación a repensar la investigación social del trabajo, con una breve revisión de nuestra propuesta, la cual apunta a ser profundizada en las experiencias de diálogo, investigación y cooperación que desarrollamos en el campo y realidad social, ante los desafíos planteados por el actual contexto de precariedad laboral.

\section{Bibliografía}

Agacino, R. 1994. Acumulación, distribución y Consensos en Chile. Disponible en: http://bibliotecadigital.academia.cl/ handle/123456789/596 (Visitado 05 de octubre de 2015).

Antúnes, R. 2011. "La nueva morfología del trabajo en Brasil. Reestructuración y precariedad", Revista Nueva Sociedad 232: 103-118.

2005 Los Sentidos del Trabajo. Ensayo sobre la afirmación y negación del trabajo. Buenos Aires: Herramienta. Taller de Estudios Laborales.

2003. ¿Adiós al Trabajo? Ensayo sobre la metamorfosis $y$ el rol central del mundo del trabajo. Buenos Aires: Herramienta. Taller de Estudios Laborales.

Antúnes, R. y Pochmann, M. 2008. "La desconstrucción del trabajo y la explosión del desempleo estructural y de la pobreza en Brasil". Producción de pobreza y desigualdad en América Latina. Cimadamore, A. y Cattani, D. (Coord.). Bogotá: Siglo del Hombre editores. 191- 204.

Aravena, A. 2007. "Reflexiones metodológicas para el studio del sindicalismo en Chile: aportes para un debate necesario". Revista Alternativa 25: 9-22.

Aravena, A. y D. Núñez. 2011. "Los Gobiernos de la Concertación y el Sindicalismo en Chile". Revista Trabajo 5 (8):
113-133.

Ariztía, T. y Bernasconi, O. 2012. "Sociologías públicas y la producción del cambio social en el Chile de los noventa". Produciendo lo Social. Usos de las ciencias sociales en el Chile reciente, Ariztía, T. (Ed.). Santiago: Ediciones Universidad Diego Portales. 133-163.

Barattini, M. 2009. "El trabajo precario en la era de la globalización ¿Es posible la Organización?" Revista Polis 24. Disponible en: http://www.redalyc.org/articulo. oa? id=30512210002

Barchiesi, F. 2011. Precarious liberation: Workers, the state, and contested social citizenship in post-apartheid South Africa. Albany: State University of New York Press.

Battistini, O. R. 2009. "La precariedad como referencial identitario: un estudio sobre la realidad del trabajo en la Argentina actual", Psicoperspectivas: Individuo y Sociedad, 8 (2): 120-142.

Bensusán, G. 2009. "Estándares laborales y calidad de los empleos en América Latina". Perfiles Latinoamericanos 34: 1349.

Bessant, J. 2008. "The sociology of policy-making in the modern state. Intent and human action". Journal of Sociology 44 (3): $283-300$. 
Blanco, O. y Julián, D. 2014. "Apuntes sobre el concepto de Modelo Productivo: estructura, formación social y conformación de subjetividades". Trabajo \& Sociedad 22: 461-478.

Boltanski, L. 2011. On critique a sociology of emancipation. Cambridge: Polity Press. XXI.

Bourdieu, P. 2008. El homo academicus. Buenos Aires: Siglo

1999. Contrafuegos. Barcelona: Anagrama.

Braga, R., Gemignani, S. y Mello, L. 2008. "Public Sociology and Social Engagement: Considerations on Brazil". Current Sociology 56 (3): 415-424.

Burawoy, M. 2014. "Introduction: Sociology as a combat sport“. Current Sociology 62: 140-155.

2012. „From Max Weber to Public Sociology”. Transnationale Vergesellschaftungen: Verhandlungen des 35. Kongresses der Deutschen Gesellschaft für Soziologie in Frankfurt am Main 2010. Soeffner, H. (ed). Frakfurt: Springer. 741-755. 2005. "Por una sociología pública". Revista Política y Sociedad 42 (1): 197-225.

2003. "For a Sociological Marxism: The Complementary Convergence of Antonio Gramsci and Karl Polanyi”. Politics \& Society 31 (2): $193-261$. 1990. "Marxism as Science: Historical Challenges and Theoretical" American Sociological Review 55 (6): 775793.

Cárdenas, A. 2005. El sujeto y el proceso de transformación en las condiciones de trabajo en Chile: Elementos teóricos $y$ empíricos para la discusión. Santiago: OIT.

Caro, P. 2012, "Magnitud y características de la participación laboral en el empleo temporal agrícola en Chile". Revista Si Somos Americanos. Estudios Transfronterizos 2 (2): 51-83.

Castel, R. 2010a. "La protección social en una sociedad de semejantes". Biblioteca Digital de la Universidad ICESI. Disponible en: http://www.icesi.edu.co/revistas/index.php/revista_cs/article/ view/400 (visitado 8 de julio de 2014)

2010b. El ascenso de las incertidumbres: trabajo, protecciones, estatuto del individuo. Buenos Aires: Fondo de Cultura Económica.

Cortina, A. 1997. Ciudadanos del mundo. Hacia una teoría de la ciudadanía. Madrid: Alianza Editorial.

De la Garza. 2012. "El trabajo no clásico y la ampliación de los conceptos de la sociología del Trabajo". Revista de Trabajo 8 (10): 109-123.

2005. "Del concepto ampliado de trabajo al de sujeto laboral ampliado". Sindicatos y nuevos movimientos sociales en América Latina. De la Garza, E. (Comp.). Buenos Aires:

Consejo Latinoamericano de ciencias sociales. 9-17 2001. "Problemas clásicos y actuales de la crisis del trabajo". El trabajo del futuro. El futuro del trabajo. De la Garza, E. y Neffa, C. (Comp.) Buenos Aires: Consejo Latinoamericano de Ciencias Sociales. 11-31.

Díaz, X., Godoy, L., Stecher, A. y J. P. Toro. 2006. Trabajo, Identidad y Vínculo Social. Reflexiones y experiencias en el capitalismo flexible. Santiago: Centro de Estudios de la Mujer.

Díaz-Salazar, R. 2004. "Trabajadores precarios. El proletariado del Siglo XXI“. Trabajadores precarios. El proletariado del Siglo XXI. Díaz-Salazar, R. (Ed.). Madrid: Ediciones HOAC. 67-108.

Dörre, K. 2009. "La Precariedad ¿Centro cuestión social del siglo XXI?". Actuel Marx Intervenciones 8: 79-108.

Escobar, P. 2000. Trabajadores y empleo en el Chile de los noventa. Santiago: LOM.

Fals Borda, O. 2009. Una sociología sentipensante para América Latina. Moncayo, V. M. (Comp). Bogotá: Siglo del Hombre Editores y CLACSO.

Gago, V. y Mezzadra, S. 2015. "Para una crítica de las operaciones extractivas del capital". Revista Nueva Sociedad 255: 38-52.

Garretón, M. A. 2014. Las ciencias sociales en la trama de Chile y América Latina. Santiago, Chile: Editorial LOM.

Góngora, J., Rodríguez, J. y Leyva, M. 2006. "Los estudios sobre el sindicalismo en América Latina y México". Revista Veredas 13 (2): 161-191.

González, P. 2009. De la sociología del poder a la sociología de la explotación: Pensar América Latina en el Siglo XXI (Antología). Bogotá: Siglo del Hombre Editores - Consejo Latinoamericano de Ciencias Sociales.

Guadamarra, R., Hualde, A. y López, S. 2012, "Precariedad Laboral y heterogeneidad ocupacional: una propuesta teóricometodológica". Revista Mexicana de Sociología 74 (2): 213-243.

Gutiérrez, F. 2014. Resistiendo en condiciones adversas. El recurso de los sindicatos chilenos a los Tribunales y a la Inspección del Trabajo (1990-2013). Ponencia Pre-ALAS y $8^{\circ}$ Congreso chileno de Sociología. La Serena, Chile: Universidad Central.

Hernández, G., Pavez, J., Rebolledo, L. y Valdés, X. 2014. Trabajos y familias en el neoliberalismo. Hombres y mujeres en la faena de la uva, el salmón y el cobre. Santiago: Editorial LOM.

Horkheimer, M. 1998. Teoría Crítica. Buenos Aires: Amorrortu.

Julián, D. 2014a. "El sindicalismo en Chile. Panorama de sus tendencias y notas para su revitalización". Revista Trabajo 8 (10): 139-167.

2014b. "Bases del modelo de valoración precario del trabajo en Chile Acercamientos desde la política laboral y la cultura del trabajo". Revista Sociológica 81 (1): 119-160.

2014c. "La precariedad laboral, modernidad y modernización capitalista: Una contribución al debate desde América Latina". Trabajo \& Sociedad 23: 147-168.

2013. "Contribución al estudio de las identidades precarias" Revista Polis 36: 407-429.

Kalleberg, A. 2011. Good jobs, bad jobs: the rise of polarized and precarious employment systems in the United States, 1970s to 2000s. Nueva York: Russell Sage Foundation.

.2009. "Precarious Work, Insecure Workers: Employment

Relations in Transition". American Sociological Review 74: 1-22.

Kalleberg, R. 2005. "What is 'public sociology'? Why and how should it be made stronger?" The British Journal of Sociology 56 (3): 387-393. 
2000. "The most important task of sociology is to strengthen and defend rationality in public discourse". On the sociology of Vilhelm Aubert'. Acta Sociologica 43 (4): 399-411.

Lechner, N. 1990. "¿Son compatibles modernidad y modernización? El desafío de la democracia latinoamericana", Documentos de Trabajo No. 440. Santiago de Chile: FLACSO-Chile.

Marini, R. M. 2007. América Latina, dependencia y globalización. (Antología). Bogotá: Siglo del Hombre Editores Consejo Latinoamericano de Ciencias Sociales.

Mayol, A. 2013. El derrumbe del modelo. Santiago: Editorial LOM.

Moulián, T. 1997. Chile actual: anatomía de un mito. Santiago, Chile: Editorial LOM.

Neffa, J.C. 2010. "La transición desde los "verdaderos empleos" al trabajo precario". Trabajo, identidad y acción colectiva. De la Garza, E. y Neffa, J.C. (Coord.). México D.F.: CLACSO - Universidad Autónoma Metropolitana Iztapalapa Plaza y Valdés. 43-80.

2009. "Sector informal, precariedad, trabajo no registrado". Ponencia en $9^{\circ}$ Congreso Nacional de Estudios del Trabajo. ASET. Buenos Aires. 5-7 de agosto. Disponible en http:// www.aset.org.ar/congresos/9/ponencias/p8_neffa.pdf

Nuñez, M. 2011. "Posibilidades y límites de la propuesta de construcción de una sociología pública”. Revista Sociológica 26 (73): 233-247.

Prashad, V. 2013. The poorer nations. A possible History of The Global South. New York: Verso.

Ramos, C. 2012. El ensamble de ciencia social y sociedad. Santiago: Editorial Universidad Alberto Hurtado.

Ramos, C., Canales, A. y Palestini, S. 2008. "El Campo de las Ciencias Sociales en Chile: ¿Convergencia disciplinar en la construcción del objeto de estudio?". Revista Cinta de Moebio 33: 171-194

Ross, A. 2008. "The new Geography of Work: Power to the Precarious?" Theory, Culture \& Society 25 (7/8): 31-49.

Salas, C. 2013. "Diálogo Social en Chile: Una mirada hacia la negociación colectiva y la fijación del salario mínimo". Revista de Filosofía y Ciencias Jurídicas 2 (2): 55-80.

Salazar, G. 2009, Del poder constituyente de asalariados e intelectuales (Chile, siglos XX y XXI). Santiago: Editorial LOM. 2008, Historia de la Acumulación de Capital en Chile. Santiago: Editorial LOM.

Sisto, V. 2012. "Identidades desafiadas: Individualización, managerialismo y trabajo docente en el Chile actual". Revista
Psykhe, 21 (2): 35-46.

2009. "Cambios en el Trabajo, Identidad e Inclusión Social en Chile: Desafíos para la Investigación". Revista Universum 24 (2): 192-216.

2005. "Flexibilización laboral de la docencia universitaria y la gest(ac)ión de la Universidad sin órganos. Un análisis desde la subjetividad laboral del docente en condiciones de precariedad." Espacio público y privatización del conocimiento Estudios sobre políticas universitarias en América Latina. Levy, B. y Gentilli, P. (Comp.). Buenos Aires: Consejo Latinoamericano de Ciencias Sociales. 523-574.

Sotelo, A. 2003. La Reestructuración del mundo del trabajo, super-explotación y nuevos paradigmas de la organización del trabajo. México, D.F.: Editorial Itaca Piraña.

Soto, Á. 2014. "Escenarios del Trabajo en Chile: Una propuesta comprensiva de la actividad laboral". Transformaciones del trabajo, subjetividad e identidades: lecturas psicosociales desde Chile y América Latina. Stecher, A. y Godoy, L. (Ed), Santiago: RIL Editores. 229-322.

2008. (Ed). 2008. Flexibilidad Laboral y Subjetividades, hacia una comprensión psicosocial del empleo contemporáneo. Santiago: LOM ediciones - Universidad Alberto Hurtado.

Standing, G. 2011. The precariat. The New Dangerous Class. London: Bloomsbury Academic.

Stecher, A. 2013. "Un modelo crítico-interpretativo para el estudio de las identidades laborales. Contribuciones a la investigación psicosocial sobre trabajo y subjetividad en América Latina". Psychologica 12 (4): 1311-1324.

Stecher, A. y L. Godoy (Ed.). 2014. Transformaciones del Trabajo, Subjetividad e Identidad. Lecturas psicosociales desde Chile y América Latina. Santiago: RIL Editores.

Van der Linden, M. 2014. "Santo Precario: A new inspiration for Labor Historians". Labor. Studies in Working-Class History of the Americas 11 (1): 9-21.

Wallerstein, I. 2006. Abrir las ciencias sociales. Informe de la Comisión Gulbenkian para la reestructuración de las ciencias sociales. México D.F.: Siglo XXI.

Weller, J. 2011. "Panorama de las condiciones de trabajo en América Latina". Nueva Sociedad No. 232: 32-49.

Zemelman, H. 2002. Necesidad de conciencia. Un modo de construir conocimiento. Barcelona: Anthropos.

(Coord.). 1995. Determinismos y Alternativas de las Ciencias Sociales Latinoamericana. Caracas: Nueva Sociedad. 